Arch. Tierz., Dummerstorf 45 (2002) 1, 87-97

Aus dem Institut für Tierzucht und Tierhaltung mit Tierklinik der Martin-Luther-Universität Halle-Wittenberg

NORBERT MIELENZ und LUTZ SCHÜLER

\title{
Nutzung von QTL-Information bei der Langzeitselektion
}

\author{
Herrn Prof. Drs. h. c. Franz Pirchner PhD, zum 75. Geburtstag gewidmet
}

\begin{abstract}
Summary
Title of the paper: Long-term selection by using QTL-information

A quantitative trait is assumed to be genetically affected by a polygenic effect and a major effect of a single dialellic locus. Such an identified gene is denoted as quantitative trait locus (QTL). A population characterized by infinite population size, heritability equal to 0.4 , ratio of QTL-variance and total genetic variance equal to $20 \%$ and complete dominance at the QTL was considered. The objective of the study was to investigate the genetic changes in the population over 20 generations of selection in five different environmental situations. The scenarios differed in the environmental variation of the three QTL-genotypes and also in heritability level after the favourable allel being fixed. To selection over generations indexes were constructed including the breeding value for the known QTL, the genotypic value of the QTL and the estimated polygenic breeding value. Beside of selection on phenotype and standard-indexes a sub-optimal index contracted the environmental variances at the QTL was considered in the model. If the favourable QTL-genotype is highly affected by the environment than it can be of advantage to select against the favourable allel. Deterministic calculations confirmed greater long-term response for sub-optimal strategies over standard indexes and phenotypic selection, not only for the up-selection but for the down-selection as well. The results from the deterministic approach showed good agreements with the results obtained by stochastic simulation.
\end{abstract}

Key Words: major gene, long-term selection, environmental variances, heterogenity

\section{Zusammenfassung}

Die Arbeit besitzt den Titel: Nutzung von QTL-Information bei der Langzeitselektion. Die genetische Fundierung der Merkmalsausprägung wird auf ein identifiziertes Einzelgen (QTL) und eine polygene Komponente zurückgeführt. Unter den Annahmen: unendliche Populationsgröße, Heritabilität gleich 0,4, Anteil der QTLVarianz an der genetischen Gesamtvarianz bei $20 \%$ und QTL unter vollständiger Dominanz wird die züchterische Bearbeitung einer fiktiven Population unter 5 verschiedenen Umweltsituationen über 20 Generationen untersucht. Die Szenarien unterscheiden sich in der Umweltvariabilität der 3 möglichen QTL-Genotypen und somit im Heritabilitätsniveau nach der Allelfixierung am QTL. Zur Selektion auf den einzelnen Generationen werden Indizes, gebildet aus dem Zuchtwert am QTL, dem genotypischen Wert am QTL und einer Schätzung für den polygenen Zuchtwert, eingesetzt. Neben der Selektion nach dem Phänotyp und Standardindexvarianten wird ein suboptimaler Index, konstruiert in Abhängigkeit von den Umweltvarianzen am QTL, in die Modellrechnungen mit einbezogen. Zeigt der gewünschte QTL-Genotyp hohe Umweltbeeinflussung, so ist es unter gewissen Bedingungen angebracht, gegen das gewünschte Allel zu selektieren. Die mit Hilfe der Downhill-Simplex-Methode entwickelten suboptimalen Indizes zeigten sowohl bei Erhöhung (Aufselektion) als auch bei Verringerung (Abselektion) der Merkmalsausprägung gegenüber den Standardvarianten teilweise erheblich höhere kumulative Selektionserfolge auf Generation 20. Die Ergebnisse, erzielt mit den deterministischen Modellrechnungen, konnten durch stochastische Simulation bei Einbeziehung von Inzucht bestätigt werden.

Schlüsselwörter: Hauptgen, Langzeitselektion, heterogene Umweltvarianzen

1. Einleitung

Die Vorhersage der genetischen Veränderung eines Merkmals infolge von Selektionsmaßnahmen ist eine zentrale Aufgabe bei der Einführung und Anwendung neuer 
Zuchtverfahren. Die klassische Theorie der Indexselektion geht von mehrdimensionaler Normalverteilung für Genotyp und Phänotyp, rein additiver Vererbung und von Umweltvarianzen, die vom genetischen Niveau der Individuen unabhängig sind, aus. In dieser Arbeit werden zur Indextheorie abweichende Voraussetzungen getroffen. Erstens wird unterstellt, dass neben einer polygenen Komponente ein identifiziertes Einzelgen mit dominantem Erbgang die Merkmalsausprägung steuert (PONG-WONG und WOOLIAMS, 1998; DECKER und VAN ARENDONK, 1998). Ein derartiges Modell wird auch als gemischtes Modell der Vererbung (GMV), der herausgehobene Genort als Hauptgenort oder QTL (Quantitative Trait Locus) und die Varianz, verursacht durch das Hauptgen, als QTL-Varianz bezeichnet. Zweitens werden unter der These, dass Individuen mit hohem genetischen Niveau sowohl weniger als auch stärker durch die Umwelt beeinflusst werden können, unterschiedliche Umweltkonstellationen für die QTL-Genotypen erzeugt. Anschließend wird die genetische Veränderung bei Langzeitselektion sowohl auf Erhöhung der Merkmalsausprägung (Aufselektion) als auch in entgegengesetzter Richtung auf Verringerung der Merkmalsausprägung (Abselektion) mit deterministischen und stochastischen Methoden untersucht. Die Selektion über 20 Generationen erfolgt nach dem Phänotyp, nach mehreren Indizes gebildet gemäß Indextheorie und nach einem suboptimalen Index konstruiert unter Beachtung der unterschiedlichen Umweltszenarien.

2. Das gemischte Modell der Vererbung

Der einfachste Fall eines gemischten Modells der Vererbung wird betrachtet, indem angenommen wird, dass die genetische Fundierung eines Merkmals auf einem identifizierten Einzelgen (QTL) und einer polygenen Komponente beruht. Das Modell besitzt die Gestalt (vgl. SIMIANER, 1995):

$$
x_{i j}=\mu_{i}+a_{i j}+u_{i j}
$$

Hierbei sind:

$\begin{array}{lll}x_{i j} & - & \text { Leistung von Tier } j \text { mit QTL-Genotyp } i \\ \mu_{i} & - & \text { genotypischer Wert von Genotyp } G_{i} \text { (phänotypisches Mittel aller Tiere } \\ & & \left.\text { mit Genotyp } G_{i}\right) \\ a_{i j} & - & \text { additiv-genetischer Effekt von Tier } j,\left(\operatorname{Var}\left(a_{i j}\right)=\sigma_{a_{i}}^{2}\right) \\ u_{i j} & - & \text { zufälliger Umwelteffekt, }\left(\operatorname{Var}\left(u_{i j}\right)=\sigma_{u_{i}}^{2}\right)\end{array}$

Weiterhin gelten die folgenden Verteilungsannahmen:

$$
x_{i j} \sim N\left(\mu_{i}, \sigma_{i}^{2}\right) \quad \text { mit } \quad E\left(x_{i j}\right)=\mu_{i} \quad \text { und } \quad \sigma_{i}^{2}=\sigma_{a_{i}}^{2}+\sigma_{u_{i}}^{2}
$$

Es soll von Hardy-Weinberg-Gleichgewicht ausgegangen werden, weiterhin seien Einzelgen und polygene Komponente voneinander unabhängig. In Modell (1) besitzen im biallelen Fall die drei möglichen Genotypen $A_{1} A_{1}, A_{1} A_{2}$ und $A_{2} A_{2}$ (oder kurz $G_{1}$, $G_{2}$ und $G_{3}$ ) die allgemeinen Ausprägungen $\mu_{1}=c+a, \mu_{2}=c+d$ und $\mu_{3}=c-a$. Bezeichne $p_{i}=\operatorname{Pr}\left(G_{i}\right)$ die Frequenz von $G_{i}$. Sei die Frequenz von Allel $A_{1}$ gleich $p$ und die von $A_{2}$ gleich $q$. Dann gilt: $p_{1}=p^{2} ; p_{2}=2 p q$ und $p_{3}=q^{2}$. Die Populationsparameter in Modell (1) besitzen (unter Beachtung, dass alle Tiere im Merkmal x einer Mischung von 3 Normalverteilungen am QTL genügen) die Darstellung: 


$$
\begin{array}{lll}
\mu=\sum_{i} p_{i} \mu_{i} \quad \text { - Populationsmittel; } & \sigma_{q}^{2}=\sum_{i} p_{i}\left(\mu_{i}-\mu\right)^{2} \text { - QTL-Varianz } \\
\sigma_{a}^{2}=\sum_{i} p_{i} \sigma_{a_{i}}^{2} \text { - polygene Varianz; } & \sigma_{u}^{2}=\sum_{i} p_{i} \sigma_{u_{i}}^{2} & \text { - Umweltvarianz }
\end{array}
$$

Weiterhin bezeichne $\sigma_{x}^{2}=\sigma_{q}^{2}+\sigma_{a}^{2}+\sigma_{u}^{2}$ die phänotypische Varianz, $\sigma_{g}^{2}=\sigma_{q}^{2}+\sigma_{a}^{2}$ die genetische Gesamtvarianz, $h^{2}=\sigma_{g}^{2} / \sigma_{x}^{2}$ die mittlere Heritabilität, $h_{p}^{2}=\sigma_{a}^{2} /\left(\sigma_{a}^{2}+\sigma_{u}^{2}\right)$ die mittlere polygene Heritabilität und $t^{2}=\sigma_{q}^{2} /\left(\sigma_{q}^{2}+\sigma_{a}^{2}\right)$ den Anteil der QTL-Varianz an der genetischen Gesamtvarianz. Die Berechnung des Langzeitselektionserfolges erfolgt deterministisch unter den Annahmen: unendliche Populationsgröße, Anpaarungsverhältnis 1:1 und Stutzungsselektion in beiden Geschlechtern. Der Formelapparat zur Berechnung der genetischen Veränderungen in Modell (1) bei Stutzungsselektion über $\mathrm{n}$ Generationen ist in der Arbeit von PONG-WONG und WOOLIAMS (1998) zu finden. Bei der Berechnung der polygenen Mittel und Varianzen in der Nachkommengeneration ist zu beachten, dass die einzelnen Elterngenotypen unterschiedliche polygene Erwartungswerte besitzen (vgl. SIMIANER, 1995). Eine ausführliche Darstellung des Formelapparates zur Berechnung der polygenen Mittel und der polygenen Varianzen auf Generation $n$ bei genereller Stutzungsselektion (inklusive Auf- und Abselektion) kann man bei MIELENZ (2000) anfordern.

\section{$2.2 \quad$ Populationscharakteristika und Umweltsituation}

Nachfolgend wird die züchterische Bearbeitung einer Population unter 5 verschiedenen Umweltsituationen betrachtet. Am QTL liege vollständige Dominanz vor. Weiterhin gelte für die Ausgangsgeneration $h^{2}=0.4 ; t^{2}=0.2$ und die Standardisierung $\mu=0$ und $\sigma_{x}^{2}=1$. Die Frequenz $p$ von $A_{1}$ sei mit 0.8 festgesetzt. Aufgrund der Vorgaben gilt $a=d=0.7217$ und $c=-0.6640$. In Anlehnung an das Problem heterogener Restvarianzen über Regionen und Unterregionen hinweg (vgl. HILL, 1984; DODENHOFF und SWALVE, 1998), seien hinsichtlich der Umweltbedingungen 5 verschiedene Situationen unterstellt. Ausgehend von der These, dass Individuen mit hohem genetischen Niveau sowohl weniger als auch stärker durch die Umwelt beeinflusst werden können, werden im Modell (1) die in Tabelle 1 aufgeführten Relationen für die Umweltvarianzen vorausgesetzt. Auf die Variation der additiv-genetischen Varianzen wurde verzichtet und nur die Umweltvarianzen drastisch ausgelenkt.

Tabelle 1

Schiefe $\left(\gamma_{1}\right)$ und Exzeß $\left(\gamma_{2}\right)$ bei Variation der Umweltvarianzen $\left(\mathrm{h}^{2}=0.4 ; \mathrm{t}^{2}=0.2\right.$ und $\left.\mathrm{p}=0.8\right)$ (Skewness and kurtosis by variation of the environmental variances)

\begin{tabular}{l|ll|lll|l|l}
\hline These & $\sigma_{a}^{2}$ & $\sigma_{q}^{2}$ & $\sigma_{u_{1}}^{2}$ & $\sigma_{u_{2}}^{2}$ & $\sigma_{u_{3}}^{2}$ & $\gamma_{1}$ & $\gamma_{2}$ \\
\hline U1 & 0.32 & 0.08 & 0.2188 & 1.2500 & 1.5000 & -0.26 & 1.32 \\
U2 & 0.32 & 0.08 & 0.2188 & 0.6875 & 6.0000 & -1.04 & 6.40 \\
U3 & 0.32 & 0.08 & 0.7500 & 0.1875 & 1.5000 & -0.26 & 0.85 \\
U4 & 0.32 & 0.08 & 0.4680 & 0.1875 & 6.0000 & -1.04 & 0.31 \\
\hline U5 & 0.32 & 0.08 & 0.6000 & 0.6000 & 0.6000 & -0.11 & 0.13 \\
\hline
\end{tabular}

Die Varianten von Tabelle 1 können als 5 Umweltszenarien interpretiert werden, unter denen oben beschriebene Population über einen Zeitraum von 20 Generationen züchterisch zu verändern ist. In allen aufgelisteten Umwelten besitzt die phänotypische 
Merkmalsausprägung den Mittelwert Null und die Varianz Eins. Allerdings führen die Umweltszenarien zu unterschiedlicher asymmetrischer Merkmalsausprägung, charakterisiert durch die Asymmetriemaße Schiefe und Exzeß (s. MIELENZ und SCHÜLER, 2000). Bei Varianzhomogenität liegt die Schiefe lediglich bei -0.11 während bei extremer Auslenkung der Verhältnisse zwischen den Umweltvarianzen gemäß Variante U2 und U4 eine Schiefe von -1.04 auftritt.

\section{3}

Selektion aufgrund von QTL-Information

Nachfolgend wird Selektion über 20 Generationen sowohl auf Erhöhung der Merkmalsausprägung (Aufselektion) als auch auf Verringerung der Merkmalsausprägung (Abselektion) untersucht. Auf jeder Generation erfolgt Stutzungsselektion nach einem Index in beiden Geschlechtern jeweils mit einer Remontierung von 20\%. Zur Rangordnung eines Tieres $j$ mit Genotyp $i$ werden Phänotyp und QTL-Information, hervorgegangen durch Typisierung, wie folgt in einen Index kombiniert:

$$
I_{i j}=b_{1 i} \cdot z w_{i}+b_{2} \cdot \mu_{i}+b_{3} \cdot\left(x_{i j}-\mu_{i}\right) ; \quad i=1, \cdots, 3
$$

Hierbei bezeichnet $z w_{i}$ die Zuchtwerte am QTL. Mit Hilfe des durchschnittlichen Effektes einer Allelsubstitution $\alpha=a+d \cdot(1-2 p)$ besitzen die 3 möglichen Zuchtwerte die Darstellung (s. FALCONER, 1984):

$$
z w_{1}=2 q \alpha ; \quad z w_{2}=(q-p) \alpha ; \quad z w_{3}=-2 p \alpha
$$

In Darstellung (2) wurde nur der Indexkoeffizient für die Zuchtwerte abhängig vom QTL-Genotyp $G_{i}$ gewählt. Soll z.B. bereits auf Generation 1 eine Fixierung des gewünschten homozygoten Genotyps bei der Aufselektion erreicht werden, so wählt man in (2) die Indexgewichte $b_{11}=b_{12}=b_{13}$ hinreichend groß, $b_{2}=0.0$ und $b_{3}$ hinreichend klein.

Die Angabe optimaler Indexgewichte für die unter (2) aufgelistete Klasse von linearen Indizes ist ein schwieriges Unterfangen. Der Zuchtwert und der Genotyp am QTL (eines zufällig aus der Population herausgegriffenen Tieres) sind jeweils diskret verteilte Zufallsgrößen, die streng genommen einer Dreipunktverteilung genügen. Die formale Anwendung der Indexkonstruktion wird möglich, falls alle Informationsquellen im Index also auch der Zuchtwert und der genotypische Effekt am QTL als stetige, unabhängig verteilte Zufallsgrößen vorausgesetzt werden (vgl. LANDE und THOMPSON, 1990). Der Zuchtwert eines Tieres ist durch $H_{1}=z w_{i}+a_{i j}$ und der genotypische Wert eines Tieres durch $H_{2}=\mu_{i}+a_{i j}$ gegeben. Sieht man $H_{1}$ bzw. $H_{2}$ als „Zuchtziel“ der Indexkonstruktion an, so ergeben sich bei formaler Aufstellung und Lösung des Indexgleichungssystems unter Verwendung mittlerer Varianzen die nachfolgend aufgeführten Indizes(vgl. SIMIANER, 1995, MALEK und DEKKER, 1998).

$$
I_{i j}^{(1)}=z w_{i}+h_{p}^{2} \cdot\left(x_{i j}-\mu_{i}\right) \quad \text { bzw. } \quad I_{i j}^{(2)}=\mu_{i}+h_{p}^{2} \cdot\left(x_{i j}-\mu_{i}\right)
$$

Die Verwendung obiger Indizes stellt nur im Fall von intermediärer Vererbung am QTL und Varianzhomogenität bei der Maximierung des Selektionserfolges für eine Generation eine optimale Strategie dar. Die optimalen Eigenschaften abgeleitet aus der Indextheorie werden um so besser erreicht, je mehr QTL's bekannt sind .Bildet man die Zuchtwerte in Index $I_{i j}^{(1)}$ von (4) mit Hilfe des Effektes einer Allelsubstitution ge- 
mäß $\alpha=a+d \cdot(1-2 p)$, wobei $p$ die aktuelle Allelfrequenz ist, so wird bei dominanter Vererbung am QTL nicht der Selektionserfolg in der nächsten Generation maximiert. Soll der Selektionserfolg auf einer Generation maximiert werden, so muss bei der Berechnung von $\alpha$ anstelle von $p$ die Frequenz $p^{*}$ der selektierten Anpaarungspartner verwendet werden (DEKKERS, 1999). Die Berechnung von $p^{*}$ erfordert also eine Vorhersage für die Allelfrequenzänderung zukünftiger Nachkommen und kann somit nur iterativ erfolgen.

Die Auswahl einer optimalen Selektionsstrategie in einem gemischten Modell der Vererbung zur Maximierung des mittel- und langfristigen Selektionserfolges stellt ein nicht triviales Optimierungsproblem dar, welches keine explizite Lösung mehr gestattet. Von DEKKERS und VAN ARENDONK (1998) wurde gezeigt, dass die Maximierung des kumulativen Selektionserfolges über mehrere Generationen auf die numerische Lösung eines optimalen Steuerproblems zurückgeführt werden kann, wobei die Autoren intermediäre Vererbung und Varianzhomogenität voraussetzen. Auf Grund dieser Tatsache werden zur züchterischen Bearbeitung der fiktiven Population in den verschiedenen Umweltsituationen 4 Standardvarianten (s. Tab. 2) und eine suboptimale Indexvariante, hervorgegangen durch Setzen von $b_{2}=0.0$ und $b_{3}=h_{p}^{2}$ bei freier Variation der Koeffizienten $b_{11}$ von $z w_{1}, b_{12}$ von $z w_{2}$ und $b_{13}$ von $z w_{3}$, betrachtet. Folglich ergab sich zur Bestimmung der Indexgewichte ein dreidimensionales Optimierungsproblem, welches mit Hilfe der Downhill-Simplex-Methode (NELDER und MEAD, 1965) gelöst wurde.

Tabelle 2

Indexvarianten unterstellt bei der Stutzungsselektion über 20 Generationen (Variants of indexes used by truncation selection over 20 generations)

\begin{tabular}{c|ccc|c|c|ll}
\hline \multirow{2}{*}{ Nr. } & \multicolumn{6}{|c|}{ Indexgewichte } & \multicolumn{2}{|c}{ Bezeichnung } \\
\cline { 2 - 6 } & $\mathrm{b}_{11}$ & $\mathrm{~b}_{12}$ & $\mathrm{~b}_{13}$ & $\mathrm{~b}_{2}$ & $\mathrm{~b}_{3}$ & \multicolumn{1}{|c}{ (Phä) } \\
2 & 0.0 & 0.0 & 0.0 & 1.0 & 1.0 & Phänotypische Selektion & $($ Geno) \\
3 & 0.0 & 0.0 & 0.0 & 1.0 & $h_{p}^{2}$ & Index mit Genotypinformation & $(\mathrm{ZW})$ \\
4 & 1.0 & 1.0 & 1.0 & 0.0 & $h_{p}^{2}$ & Index mit Zuchtwertinformation & (Poly) \\
\hline
\end{tabular}

Die Varianten 2 und 3 in obiger Tabelle fallen bei rein additiver Genwirkung am QTL zusammen. Der 3. Index in Tabelle 2 wird in der Literatur auch als „standard“ MAS, wobei MAS für Marker-Assisted Selektion steht, bezeichnet (s. MALEK and DEKKERS, 1998). Die Klasse der unter (2) vorgestellten Indizes kann erweitert werden, indem z.B. $b_{3}$ vom QTL-Genotyp abhängig gewählt wird. Setzt man $b_{3 i}=h_{p_{i}}^{2}$ (mit $h_{p_{i}}^{2}$ polygene Heritabilität für alle Tiere mit Genotyp $G_{i}$ ), dann liefert die Variante 4 eine rein polygene Selektion. Derartige Varianten wurden untersucht. Sie blieben aber im Selektionserfolg hinter den im Abschnitt 3 vorgestellten suboptimalen Indizes zurück.

3. Ergebnisse

Der kumulative Selektionserfolg nach 20 Generationen $\left(\mathrm{SE}_{20}\right)$, die Frequenz des gewünschten Allels auf Generation 1 und 20 sowie die Generation der Fixierung (Frequenz des gewünschten Allels größer als 0.99) sind für die Selektion nach Phänotyp 
und den suboptimalen Index in den Tabellen 3 und 4 angegeben. Die aufgeführten suboptimalen Indizes sind für die (gemäß (2)) gewählte Klasse von Indizes optimal. Sie werden jedoch als suboptimal bezeichnet, weil z.B. b ${ }_{3}$ vom QTL-Genotyp abhängig sein kann und auf jeder Generation andere Indizes angewendet werden können. In den hier durchgeführten Modellrechnungen waren 3 Indexgewichte frei wählbar. Erweitert man die Klasse der Indizes, so kann sich die Dimension bei der Optimierung des Selektionserfolges nach 20 Generationen erheblich erhöhen wobei mehrere optimale Selektionsstrategien denkbar sind.

In den Ergebnisstabellen 3 und 4 wird generell eine mittlere Heritabilität von 0,4 vorausgesetzt. Ist der Anteil der QTL-Varianz an der genetischen Gesamtvarianz 0,2, so folgt bei einer phänotypischen Varianz von 1,0 für die QTL-Varianz ein Wert von 0,08 und für die mittlere polygene Heritabilität ein Wert von 0,32.

\subsection{Aufselektion}

Tabelle 3 veranschaulicht die genetischen Veränderungen in der Population bei Aufselektion unter den Umweltsituationen U1 bis U5. Der Selektion ausschließlich nach Phänotyp, Vektor der Indexgewichte $b=(0,0,0,0,1,1)$ ' ist die kombinierte Selektion nach einem suboptimalen Index aus Zuchtwert und polygener Komponente gegenübergestellt. Die phänotypische Selektion führt unter U1 und U2 zu keiner Fixierung eines Allels (in Tabelle 3 gekennzeichnet durch das mathematische Symbol für unendlich). Unter den Umweltszenarien U1 und U2 besitzt der bei Aufselektion gewünschte QTL-Genotyp $A_{1} A_{1}$ (genotypischer Wert $\mu_{1}=c+a$ ) auch gleichzeitig mit 0.22 die geringste Umweltbeeinflussbarkeit. Deshalb ist es von Vorteil schon auf Generation Eins das Allel $A_{1}$ zu fixieren. Bei der Selektion auf den nachfolgenden Generationen kann dann die höhere Heritabilität für den Genotyp $A_{1} A_{1}$ zusätzlich ausgenutzt werden.

Die Wahl $b_{11}=b_{12}=b_{13}=10.0$ bedingt zuerst eine Vorselektion aller Tiere mit Genotyp $A_{1} A_{1}$ (wegen $\mathrm{p}=0.8$ besitzen $64 \%$ der Tiere diesen Genotyp), wobei anschließend aus diesen Tieren die $20 \%$ besten nach der Komponente $\left(x_{i j}-\mu_{i}\right)$ endgültig zur Erzeugung der Nachkommenschaft ausgewählt werden. Hinsichtlich des polygenen Selektionserfolges wird also nur noch eine Remontierung von 31.25\% realisiert. Die Angabe einer optimalen Selektionsstrategie für die Umweltvarianten U3 und U4 erweist sich als schwierig, weil jetzt der heterozygote Genotyp $A_{1} A_{2}$ die geringste Umweltvariabilität zeigt. Durch Maximierung mit der Downhill-Simplex-Methode wurde ein suboptimaler Index aus Zuchtwert und geschätzter polygener Komponente bestimmt, der die Frequenz von $A_{1}$ in etwa auf dem Ausgangsniveau von 0.8 belässt bzw. ein leichtes Absinken bewirkt. So konnte mit der Wichtung $b_{11}=0.432 ; b_{12}=-1.350$ und $b_{13}=0.005$ für Umweltvariante U3 der Selektionserfolg von 7.68 auf 8.49 erhöht werden. In Umweltvariante U5 besitzen alle 3 Genotypen identische Umweltvarianzen. Eine Indexstrategie, welche zu einem stetigen Anstieg der Frequenz des gewünschten Allels über den gesamten Selektionszeitraum führt, wobei auf den ersten Generationen der Anstieg nur gering sein darf, scheint einer optimalen Vorgehensweise nahe zu kommen. 
Tabelle 3

Ergebnisse der Aufselektion für den Phänotyp (1. Zeile innerhalb Umweltvariante) und den suboptimalen Index (2. Zeile innerhalb Umweltvariante) (mit $h^{2}=0.4, t^{2}=0.2$ und $p=0.8$ ) (Results of up-selection on phenotype and on a sub-optimal index)

\begin{tabular}{|c|c|c|c|c|c|c|c|c|c|c|c|c|}
\hline \multirow[b]{2}{*}{ Nr.: } & \multicolumn{3}{|c|}{ Umweltvarianzen } & \multicolumn{5}{|c|}{ Indexgewichte } & \multirow[b]{2}{*}{$S E_{20}$} & \multicolumn{2}{|c|}{ Freq. von $A_{1}$} & \multirow{2}{*}{$p>0.99$} \\
\hline & $\sigma_{u_{1}}^{2}$ & $\sigma_{u_{2}}^{2}$ & $\sigma_{u_{3}}^{2}$ & $b_{11}$ & $b_{12}$ & $b_{13}$ & $b_{2}$ & $b_{3}$ & & $p_{1}$ & $p_{20}$ & \\
\hline \multirow{2}{*}{ U1 } & \multirow{2}{*}{0.22} & \multirow{2}{*}{1.25} & \multirow{2}{*}{1.50} & 0.0 & 0.0 & 0.0 & 1.0 & 1.0 & 8.39 & 0.76 & 0.72 & $\infty$ \\
\hline & & & & 10.0 & 10.0 & 10.0 & 0.0 & $h_{p}^{2}$ & 9.69 & 1.00 & 1.00 & 1 \\
\hline \multirow{2}{*}{ U2 } & \multirow{2}{*}{0.22} & \multirow{2}{*}{0.69} & \multirow{2}{*}{6.00} & 0.0 & 0.0 & 0.0 & 1.0 & 1.0 & 8.34 & 0.76 & 0.61 & $\infty$ \\
\hline & & & & 10.0 & 10.0 & 10.0 & 0.0 & $h_{p}^{2}$ & 9.69 & 1.00 & 1.00 & 1 \\
\hline \multirow[b]{2}{*}{ U3 } & \multirow[b]{2}{*}{0.75} & \multirow[b]{2}{*}{0.19} & \multirow[b]{2}{*}{1.50} & 0.0 & 0.0 & 0.0 & 1.0 & 1.0 & 7.68 & 0.87 & 1.00 & 12 \\
\hline & & & & 0.432 & -1.350 & 0.005 & 0.0 & $h_{p}^{2}$ & 8.49 & 0.75 & 0.68 & $\infty$ \\
\hline \multirow{2}{*}{ U4 } & \multirow{2}{*}{0.47} & \multirow{2}{*}{0.19} & \multirow{2}{*}{6.00} & 0.0 & 0.0 & 0.0 & 1.0 & 1.0 & 8.66 & 0.83 & 0.98 & 26 \\
\hline & & & & 1.429 & -1.365 & 0.832 & 0.0 & $h_{p}^{2}$ & 8.86 & 0.79 & 0.79 & $\infty$ \\
\hline \multirow{2}{*}{ U5 } & \multirow{2}{*}{0.60} & \multirow{2}{*}{0.60} & \multirow{2}{*}{0.60} & 0.0 & 0.0 & 0.0 & 1.0 & 1.0 & 7.96 & 0.83 & 0.96 & 103 \\
\hline & & & & -0.523 & 0.401 & 0.221 & 0.0 & $h_{p}^{2}$ & 7.98 & 0.81 & 0.96 & 63 \\
\hline
\end{tabular}

\subsection{Abselektion}

Betrachten wir nun die genetischen Veränderungen bei der Abselektion. Die phänotypische Selektion führt unter allen Umweltvarianten zu einer Fixierung von Allel $A_{2}$ nach 5, 6 oder 7 Generationen. Ursache ist die hohe Umweltvarianz des bei der Abselektion bevorzugten Genotyps $A_{2} A_{2}$ und die damit verbundene bessere Trennung der restlichen Genotypen schon bei der einfachen phänotypischen Selektion. Eine schnelle Fixierung ist hinsichtlich des Langzeitselektionserfolges nicht angeraten, weil der bevorzugte Genotyp $A_{2} A_{2}$ (mit dem genotypischen Wert $\mu_{3}=c-a$ ) hohe Umweltvariabilität zeigt und somit geringeres $\mathrm{h}^{2}$-Niveau in den nachfolgenden Generationen besitzt. Unter den Umweltkonstellationen U1 und U2 erweist es sich sogar als optimal gegen das gewünschte Allel zu selektieren. Fixiert man den unterlegenen Genotyp $A_{1} A_{1}$ bereits auf Generation Eins und nutzt dessen erhöhte Heritabilität bei der phänotypischen Selektion in den verbleibenden 19 Generationen, so erhöht sich der absolute Selektionserfolg von 7.29 auf 9.57 in Umwelt 1 bzw. von 5.14 auf 9.57 in Umwelt 2.

Unter den Konstellationen U3 bis U5 lässt sich ebenfalls der Selektionserfolg aus phänotypischer Selektion durch Indexbildung erheblich verbessern. Suboptimale Selektionsstrategien erhält man, indem die Indexkoeffizienten für die Zuchtwerte sämtlich kleiner als Eins gewählt werden, so dass ein stetiger Anstieg der Frequenz von $A_{2}$ bei Fixierung möglichst erst in Generation 20 erreicht wird. Bei Variante U4 erwies sich ein nur moderater Anstieg der Frequenz von $A_{2}$ als günstig, um die höheren Heritabilitäten der negativ besetzten Genotypen möglichst über den gesamten Selektionszeitraum nutzen zu können. 
Tabelle 4

Ergebnisse der Abselektion für den Phänotyp (1. Zeile innerhalb Umweltvariante) und den suboptimalen Index (2. Zeile innerhalb Umweltvariante) (mit $h^{2}=0.4, t^{2}=0.2$ und $q=0.2$ ) (Results of down-selection on phenotype and on a sup-optimal index)

\begin{tabular}{|c|c|c|c|c|c|c|c|c|c|c|c|c|}
\hline \multirow[b]{2}{*}{ Nr.: } & \multicolumn{3}{|c|}{ Umweltvarianzen } & \multicolumn{5}{|c|}{ Indexgewichte } & \multirow[b]{2}{*}{$S E_{20}$} & \multicolumn{2}{|c|}{ Freq. von $A_{2}$} & \multirow[b]{2}{*}{$\mathrm{q}>0.99$} \\
\hline & $\sigma_{u_{1}}^{2}$ & $\sigma_{u_{2}}^{2}$ & $\sigma_{u_{3}}^{2}$ & $b_{11}$ & $b_{12}$ & $b_{13}$ & $b_{2}$ & $b_{3}$ & & $q_{1}$ & $q_{20}$ & \\
\hline \multirow{2}{*}{ U1 } & \multirow{2}{*}{0.22} & \multirow{2}{*}{1.25} & \multirow{2}{*}{1.50} & 0.0 & 0.0 & 0.0 & $\overline{1.0}$ & 1.0 & -7.29 & 0.35 & 1.00 & 5 \\
\hline & & & & -10.0 & -10.0 & -10.0 & 0.0 & $h_{p}^{2}$ & -9.57 & 0.00 & 0.00 & $\infty$ \\
\hline \multirow[b]{2}{*}{ U2 } & \multirow[b]{2}{*}{0.22} & \multirow[b]{2}{*}{0.69} & \multirow[b]{2}{*}{6.00} & 0.0 & 0.0 & 0.0 & 1.0 & 1.0 & -5.14 & 0.31 & 1.00 & 5 \\
\hline & & & & -10.0 & -10.0 & -10.0 & 0.0 & $h_{p}^{2}$ & -9.57 & 0.00 & 0.00 & $\infty$ \\
\hline \multirow{2}{*}{ U3 } & \multirow{2}{*}{0.75} & \multirow{2}{*}{0.19} & \multirow{2}{*}{1.50} & 0.0 & 0.0 & 0.0 & 1.0 & 1.0 & -7.52 & 0.23 & 1.00 & 7 \\
\hline & & & & 0.274 & 0.450 & -0.297 & 0.0 & $h_{p}^{2}$ & -9.51 & 0.21 & 0.99 & 21 \\
\hline \multirow[b]{2}{*}{$\mathrm{U} 4$} & \multirow[b]{2}{*}{0.47} & \multirow[b]{2}{*}{0.19} & \multirow[b]{2}{*}{6.00} & 0.0 & 0.0 & 0.0 & 1.0 & 1.0 & -5.61 & 0.24 & 1.00 & 7 \\
\hline & & & & 0.337 & -0.172 & -0.217 & 0.0 & $h_{p}^{2}$ & -9.41 & 0.20 & 0.87 & 24 \\
\hline \multirow{2}{*}{ U5 } & \multirow{2}{*}{0.60} & \multirow{2}{*}{0.60} & \multirow{2}{*}{0.60} & 0.0 & 0.0 & 0.0 & 1.0 & 1.0 & -8.93 & 0.29 & 1.00 & 6 \\
\hline & & & & 0.130 & 0.149 & 0.050 & 0.0 & $h_{p}^{2}$ & -9.22 & 0.22 & 1.00 & 18 \\
\hline
\end{tabular}

\subsection{Vergleich der Auf- und Abselektion}

Eine Gegenüberstellung verschiedener Indexvarianten gemäß Tabelle 1 unter Einbeziehung der durch Variantenrechnung bestimmten suboptimalen Indizes (aufgelistet in den Tabellen 3 und 4) hinsichtlich der zwei Selektionsrichtungen enthält Tabelle 5. Der jeweilige suboptimale Selektionsindex, ermittelt unter Beachtung der konkreten Umweltsituation, zeigt sich der rein phänotypischen Selektion und den Standardindexvarianten teilweise erheblich überlegen. Bei der Abselektion lassen sich im Durchschnitt über alle Umweltszenarien höhere Selektionserfolge relativ zur Aufselektion realisieren. Hier kommt die Tatsache zum Ausdruck, dass die Frequenz von Allel $A_{2}$ zu Beginn der Selektion mit 0.2 angesetzt wurde, so dass über einen längeren Zeitraum Variabilität am QTL zur Verfügung steht.

Tabelle 5

Kumulativer Selektionserfolg nach 20 Generationen bei Einsatz unterschiedlicher Selektionsmethoden (Cumulative response to selection over 20 generations by using different selection methods)

\begin{tabular}{c|cccc|c|cccc|c}
\hline & \multicolumn{4}{|c|}{ Aufselektion } & \multicolumn{5}{c}{ Abselektion } \\
\cline { 2 - 11 } Nr. & Phä & Poly & ZW & Geno & Sub & Phä & Poly & ZW & Geno & Sub \\
\hline U1 & 8.39 & 5.64 & 9.45 & 8,57 & 9,69 & -7.29 & -7.86 & -7.17 & -7.05 & -9.57 \\
U2 & 8.34 & 3.23 & 9.60 & 9.15 & 9.69 & -5.14 & -6.00 & -4.91 & -4.83 & -9.57 \\
U3 & 7.68 & 7.80 & 7.53 & 7.66 & 8.49 & -7.52 & -7.68 & -7.35 & -6.99 & -9.51 \\
U4 & 8.66 & 8.81 & 8.47 & 8.60 & 8.86 & -5.61 & -8.76 & -5.06 & -4.90 & -9.41 \\
U5 & 7.96 & 7.93 & 7.95 & 7.96 & 7.98 & -8.93 & -7.93 & -8.94 & -8.71 & -9.22 \\
\hline
\end{tabular}

Die Selektion nach dem Phänotyp zeigt im Vergleich der Auf- und Abselektion in den Umwelten U1 bis U4 deutliche asymmetrische Selektionserfolge.

Auf die Ableitung optimaler Indexgewichte wurde verzichtet. DEKKERS und VAN ARENDONK (1998) zeigen für den Fall eines QTL unter intermediärer Vererbung bei homogener Umweltvarianz, dass die optimalen Indexgewichte für den Zuchtwert abhängig von der Generationsnummer gewählt werden müssen. Schon für den oben beschriebenen einfachen Fall muss für die Bestimmung der Indexgewichte ein nichtline- 
ares Optimierungsproblem aus der Steuertheorie gelöst werden. Deshalb wurde auf die Berechnung generationsabhängiger Indexgewichte verzichtet.

\subsection{Vergleich mit stochastischer Simulation}

Den Ergebnissen aus Abschnitt 3.1 bis 3.3 liegt unendliche Populationsgröße bei Vernachlässigung von Inzucht zugrunde. Um die Vorhersagen für den Langzeitselektionserfolg mit dem deterministischen Modell zu überprüfen, wurde zusätzlich die stochastische Simulation eingesetzt (vgl. PONG-WONG, WOOLIAMS, 1998 und MALEK, DEKKERS, 1998). Die simulierte Population wurde mit 20 Elternpaaren und 10 Nachkommen je Paar auf jeder Generation erzeugt. Folglich standen auf jeder Generation 100 weibliche und 100 männliche Tiere zur Verfügung, aus denen gemäß dem Index (2) die 20\% besten Tiere zur Erzeugung der nächsten Generation ausgewählt wurden. Nach Abschluss der Selektion lag der mittlere Inzuchtkoeffizient in der Population zwischen 25 und 30\%.

Tabelle 6

Ergebnisse der stochastischen Simulation bei Abselektion (20 Elternpaare, 10 Nachkommen pro Paar, Zufallspaarung) (Results of stochastic simulation by down-selection)

\begin{tabular}{c|ccc|c|c|c|c|c|c|c}
\hline \multirow{2}{*}{ Nr.: } & \multicolumn{2}{|c}{ Suboptimale Indexgew. } & \multicolumn{3}{c|}{ Sub } & \multicolumn{3}{c}{ Phä } \\
\cline { 2 - 11 } & $\mathbf{b}_{\mathbf{1 1}}$ & $\mathbf{b}_{\mathbf{1 2}}$ & $\mathbf{b}_{\mathbf{1 3}}$ & $\mathbf{q}_{\mathbf{1}}$ & $\mathbf{q}_{\mathbf{2 0}}$ & $\mathbf{S E}_{\mathbf{2 0}}$ & $\mathbf{q}_{\mathbf{1}}$ & $\mathbf{q}_{\mathbf{2 0}}$ & $\mathbf{S E}_{\mathbf{2 0}}$ \\
\hline $\mathbf{U 1}$ & -10.00 & -10.00 & -10.00 & 0.00 & 0.00 & -8.41 & 0.34 & 1.00 & -6.57 \\
$\mathbf{U 2}$ & -10.00 & -10.00 & -10.00 & 0.00 & 0.00 & -8.41 & 0.31 & 1.00 & -4.96 \\
$\mathbf{U 3}$ & 0.274 & 0.450 & -0.297 & 0.21 & 0.39 & -7.66 & 0.22 & 0.71 & -6.56 \\
$\mathbf{U} 4$ & 0.337 & -0.172 & -0.217 & 0.22 & 0.54 & -7.19 & 0.24 & 0.83 & -5.70 \\
$\mathbf{U 5}$ & 0.300 & 0.149 & 0.050 & 0.25 & 0.96 & -8.16 & 0.28 & 1.00 & -7.84 \\
\hline
\end{tabular}

Resultate der Modellrechnungen mit stochastischer Simulation basierend auf 200 Wiederholungen sind für den Fall der Abselektion in Tabelle 6 enthalten. Der suboptimale Indexkoeffizient $b_{11}$ musste im Vergleich zu dem entsprechenden Wert aus Tabelle 4 bei der Variante U5 erheblich vergrößert werden, um einen stetigen Anstieg der Frequenz des gewünschten Allels $\mathrm{A}_{2}$ mit Fixierung nahe Generation $20 \mathrm{zu}$ gewährleisten. Bei den Varianten U3 und U4 erwies sich der für unendliche Populationsgröße bestimmte Index der phänotypischen Selektion auch bei der Simulation überlegen. Für die Frequenz von $\mathrm{A}_{2}$ resultierte in Variante U3 bzw. U4 ein nur moderater Anstieg bis zu einem Wert von 0.39 bzw. von 0.54, so dass eine weitere Optimierung der Indexgewichte möglich sein sollte. Zwischen den kumulativen Selektionserfolgen nach 20 Generationen für den suboptimalen Index und für die Selektion nach Phänotyp ergaben sich die erwarteten Relationen. Aus Rechenzeitgründen wurde darauf verzichtet alle Indexkoeffizienten an die Populationsgröße und die davon abhängige Inzuchtentwicklung neu anzupassen. Es sollte lediglich gezeigt werden, dass unter Einschluss von QTL-Information Indizes existieren, die der einfachen phänotypischen Selektion im Langzeitselektionserfolg erheblich überlegen sind.

4. Diskussion

Die effektive züchterische Bearbeitung einer Population über einen längeren Zeitraum bei Vorhandensein von QTL- und phänotypischer Information setzt zusätzlich Kenntnisse über die Umweltvariabilität der QTL-Genotypen voraus. Bei der Fixierung eines 
QTL’s ist die Umweltvariabilität des bevorzugten gewünschten QTL-Genotyps zu beachten.

Die für die Zuchtarbeit günstigste Situation liegt vor, falls der gewünschte QTL-Genotyp auch die geringste Umweltvariation zeigt. Eine kurzfristige Fixierung des gewünschten QTL-Allels ist zu vermeiden, falls der zugehörige Genotyp hohe Umweltvariabilität aufweist, so dass die weitere züchterische Bearbeitung der Population auf niedrigem $\mathrm{h}^{2}$-Niveau erfolgen muss.

Eine Fixierung kann sich auch als ungünstig erweisen, falls der bevorzugte QTL-Genotyp unter veränderten Umweltbedingungen stärker als die von der Weiterzucht ausgeschlossenen QTL-Genotypen beeinflusst wird.

Die züchterisch anspruchvollste Situation an eine langfristige Selektionsstrategie liegt vor, falls der gewünschte homozygote Genotyp die höchste Umweltbeeinflussung besitzt und zusätzlich der heterozygote Genotyp an die geringste Umweltvariation gekoppelt ist.

In den Untersuchungen konnte gezeigt werden, dass durch geeignete Kombination von QTL- und phänotypischer Information eine wesentliche Steigerung des Langzeitselektionserfolges erreicht werden kann. Allerdings unter der Annahme, dass sich die Umweltwirkungen zu Beginn der Selektion quantifizieren lassen und über den Selektionszeitraum hinweg konstant bleiben.

Sowohl das deterministische Modell als auch die stochastische Simulation können zur Auffindung von zumindest suboptimaler Indizes für die Langzeitselektion genutzt werden. Mit dem deterministischen Ansatz sind in einem ersten Schritt Vorstellungen über die Selektionsstrategie zu entwickeln. Im zweiten Schritt erfolgt dann unter Verwendung der stochastischen Simulation die Abstimmung der abgeleiteten Indexgewichte auf den konkreten Populationsumfang und das Verpaarungsschema. Die hier beschriebene Vorgehensweise erfordert eine klare genetische Charakterisierung der Ausgangspopulation und zusätzlich Kenntnisse über die Umweltbedingungen, in denen die Population über einen längeren Zeitraum züchterisch zu bearbeiten ist.

\section{Literatur}

DEKKERS, J.C.M.; VAN ARENDONK, J.A.M.:

Optimum selection on identified quantitative trait loci. Proceedings of the $6^{\text {th }}$ World Congress on Genetics Applied to Livestock Production. Armidale Australia, 26 (1998), 361-364

DEKKERS, J.C.M.:

Breeding values for identified quantitative trait loci under selection. Genetics, Selection, Evolution 31 (1999) (5/6), 421-436

DODENHOFF, J.; SWALVE, H.H.:

Heterogeneity of variances across regions of northern Germany and adjustment in genetic evaluation. Livestock Production Science 53 (1998), 225-236

FALCONER, D.S.:

Einführung in die Quantitative Genetik. (Übersetzung von GLODEK, P.) Eugen Ulmer (1984) Stuttgart

HILL, W.G.:

On selection among groups with heterogeneous variance. Animal Production 39 (1984), 473-477

LANDE, R.; THOMPSON, R.:

Efficiency of marker-assisted selection in the improvement of quantitative Einführung in die Quantitative Genetik. (Übersetzung von GLODEK, P.) Eugen Ulmer (1984) Stuttgart Traits. Genetics 124 (1990), 743-756

MALEK, M.; DEKKERS, J.C.M.:

A simulation study to evaluate optimal strategies for selection on a quantitative trait using major gene information. J. Anim. Sci. 76 (1998), Suppl.1 
MIELENZ, N.; SCHÜLER, L.:

Auswirkungen eines QTL auf die Asymmetrie der Merkmalsausprägung. Arch. Tierz., Dummerstorf 41(1998) 4, 407-414

MIELENZ, N.; SCHÜLER, L.:

Langzeitselektion bei asymmetrischer Merkmalsverteilung. Arch. Tierz., Dummerstorf 43 (2000) 5, 421-430

MIELENZ, N.:

Berechnung des Selektionserfolges bei Stutzungsselektion in einem gemischten Modell der Vererbung. Unveröffentlichtes Manuskript (2000)

NELDER, J.A.; MEAD, R.:

A simplex method for function minimization. Computer Journal, 7 (1965), 308-313

PONG-WONG, R.; WOOLLIAMS, J.A.:

Response to mass selection when an identified major gene is segregating. Genet. Sel. Evol. 30 (1998), 313-337

SIMIANER, H.:

Manuskript zum Kurs „, Markergestützte Selektion in der Tierzucht - Künstliche Selektion unter verschiedenen genetischen Modellen“, Universität Hohenheim 13.-17. November (1995)

Eingegangen: 29.06.2001

Akzeptiert: 07.12.2001

Anschrift der Verfasser

Dr. NORBERT MIELENZ, Univ.-Prof. Dr. habil. LUTZ SCHÜLER

Institut für Tierzucht und Tierhaltung mit Tierklinik der

Martin-Luther-Universität Halle-Wittenberg

Adam-Kuckhoff-Straße 35

D-06108 Halle

E-Mail: mielenz@landw.uni-halle.de, schueler@landw.uni-halle.de 